EXEMPLARIa Classica

Journal of Classical Philology

18, 2014, pp. 99-104

ISSN 1699-3225

\title{
CHARISIANA IV: ¿INAVRATAE O INAVRATE? VAIVENES DE UNA VARIANTE (CHAR. GRAMM. P. 262, 27-8)*
}

\author{
JAVIER URÍA VARELA \\ Universidad de Zaragoza \\ juria@unizar.es \\ Marcos Antonio Pérez Alonso \\ mperez931@hotmail.com
}

\section{SUMMARY}

This paper deals with some shocking nominal items in a list of adverbs in Charisius' Ars grammatica. We pay particular attention to the lemma inaurate, subject to controversy from the beginning of the textual transmission. We provide a coherent picture in terms of both textual criticism and the grammatical tradition.

KEYWORDS

Charisius and Iulius Romanus, adverbs, textual criticism.

\section{RESUMEN}

Este artículo trata algunas extrañas entradas nominales de una lista de adverbios del Ars grammatica de Carisio. Prestamos particular atención al lema inaurate, objeto de debate desde el comienzo de la transmisión textual. Proporcionamos un cuadro coherente tanto en relación con la crítica textual como con la tradición gramatical.

Palabras clave

Carisio y Julio Romano, adverbios, crítica textual.

Fecha de recepción: 23/05/2014

Fecha de aceptación y versión final: 17/09/2014

"Este artículo supone una extensión de la contribución de J. Uría (Nomen an adverbium? Latin Grammarians on the Adverb) al congreso Il latino dei grammatici, Pisa 2013), y es resultado de la discusión de los dos coautores sobre aspectos concretos relacionados con dicha contribución. Ha sido realizada en el marco del Proyecto de Investigación FFI201130203-C02-02 del Ministerio de Economía y Competitividad (España). Los coautores agradecen las observaciones de los revisores anónimos. 
El gramático del siglo III Julio Romano es conocido fundamentalmente por el uso que de su obra gramatical hizo, a finales del s. IV, Flavio Sosípatro Carisio. Junto a algunas secciones abreviadas sobre preposición, interjección y conjunción, destacan las largas secciones sobre la analogía y el adverbio. En esta última (ocupa las páginas 246-89 en la edición de Barwick), tras una interesantísima introducción (en las páginas 246-52), el cuerpo principal lo forma una lista alfabética de entradas, que ha despertado más interés por los ejemplos que la acompañan -con frecuencia testimonios únicos de fragmentos de autores arcaicos- que por las entradas en sí. Entre éstas, sin embargo, algunas tienen interés intrínseco, porque ponen de relieve la difusa definición que el adverbio tuvo ya en la gramática antigua; nos referimos sobre todo a aquellas cuya evidente naturaleza nominal hace sorprendente su inclusión en una lista de adverbios ${ }^{1}$. Es el caso, por ejemplo, de la entrada primus (Char. gramm. p. 272, 25), ejemplificada con el virgiliano Troiae qui primus ab oris. La clasificación de primus como adverbio responde a lo que en otro lugar hemos denominado regla de conmutación, según la cual, para los gramáticos latinos, puede ser adverbio cualquier elemento que se pueda sustituir por un adverbio o un sintagma adverbial ${ }^{2}$. Otra regla justifica la inclusión entre los adverbios, por ejemplo, de los usos lativos, ablativos y locativos de los nombres de ciudad, o de ciertas formas del gerundio y el supino ${ }^{3}$.

En cambio, el recurso a doctrinas gramaticales antiguas no parece, a primera vista, explicar la inclusión en la lista de adverbios de Romano de otras unidades indiscutiblemente nominales. Es el caso de la que querríamos discutir aquí, a saber, la entrada que nos proporciona un fragmento de Titinio (Titin. 1 Ribbeck inauratae atque inlautae mulieris) en el que el elemento ejemplificado (inauratae) es, a todas luces, un adjetivo concordado con mulieris. A pesar de esa evidencia, los editores de Carisio se han dividido entre imprimir el título de la entrada como inauratae, en coherencia con el ejemplo, pero no con la lista de adverbios, o como inaurate, dando prioridad a que la entrada sea un verdadero adverbio y no un adjetivo. Al final del artículo daremos una razonable $-\mathrm{y}$, a nuestro juicio, impecable- explicación de la incoherencia, y defenderemos inauratae como lectura correcta. Pero antes haremos un repaso de la larga historia de dudas que han generado estas dos líneas del ars grammatica de Carisio: una historia que empezó hace trece siglos, como pronto veremos.

${ }^{1}$ Sobre su tipología y las causas de su clasificación como adverbios véase la contribución mencionada en la nota inicial (Uría, en prensa).

${ }^{2}$ En este caso, primus uenit equivaldría a in primis uenit. Es sabido que la función de predicativo la comparten en muchas lenguas el nombre y el adverbio. Sobre la regla de conmutación véase, con más amplitud, Uría, en prensa.

${ }^{3}$ Se trata de la regla de aversión a la preposición, algo característico del adverbio según la gramática antigua (Char. gramm. p. 149,13-4 Praecipue et hoc obseruandum est in primis quod numquam aduerbium praepositionem recipiat). Véase igualmente Uría, en prensa. 
Vaya por delante el texto actualmente canónico (el editado por Keil y Barwick $^{4}$ ), que reproducimos aquí con el aparato crítico de Keil, preferible al de Barwick por razones que más abajo apuntamos:

Roman.-Char. gramm. p. 262, 27-8 Barwick (= I 202,16-7 Keil) Inaurate pro non aurate Titinius in barrato, 'inauratae atque inlautae mulieris'

inauratę (corr. -te) pro non auratę (corr. -te) $N$ : inaurate pro non aurate excerpta Bernensia

Además del paralelo de los Excerpta Bernensia mencionado por Keil y Barwick ${ }^{5}$, podemos añadir el aún más reducido (sólo el lema, bajo la forma inaurate) que proporciona el Parisinus Latinus $7530^{6}$. En realidad, estos excerpta no hacen otra cosa que anticiparse a los editores más recientes en el intento de salvar la dificultad que entraña la presencia de un adjetivo como entrada en una lista de adverbios: caen, eso sí, en otra contradicción, la que surge entre lema y ejemplo, adverbial aquél (inaurate), nominal éste (inauratae).

Lo que resulta aún más interesante $-\mathrm{y}$ lo que probablemente convenció a Keil y a Barwick de que la lectura correcta tenía que ser inaurate- es que el propio Neapolitanus, aunque seguramente de forma independiente de los mencionados excerpta $a^{7}$, señalaba con un punto de expunción ${ }^{8}$ supra lineam la conveniencia de suprimir la penúltima letra (una a) tanto de inauratae como de auratae .

${ }^{4}$ H. Keil, Grammatici Latini, Hildesheim 1961 (= 1857); K. Barwick, Flavii Sosipatri Charisii artis grammaticae libri $V$ (addenda et corrigenda collegit et adiecit F. Kühnert), Leipzig $1964^{2}\left(1925^{1}\right)$.

${ }^{5}$ El manuscrito en cuestión (Bernensis 123) reproduce una lista de los lemas, ya sea con sólo su encabezamiento o en una versión muy sucinta. Véase la transcripción de H. Hagen, Anecdota Helvetica quae ad Grammaticam Latinam spectant ex bibliothecis Turicensi Einsidlensi Bernensi, Leipzig 1870, CLIX-CLXI.

${ }^{6} \mathrm{El}$ lema que nos ocupa aparece concretamente entre las líneas 21 y 22 del verso del folio 120. El manuscrito puede consultarse en Gallica (gallica.bnf.fr), y ha sido estudiado a fondo por L. Holtz, "Le Parisinus Latinus 7530, synthèse cassinienne des arts libéraux", Studi Medievali $16,1975,97-152$.

${ }^{7}$ El Neapolitanus IV.A.8 representa una tradición diferente, aunque procedente del mismo arquetipo, a la del Parisinus Latinus 7530 (Barwick, Flavii Sosipatri, xI; M. De Nonno, La grammatica dell' "Anonymus Bobiensis” (GL I 533-565 Keil). Con un'appendice carisiana, Roma 1982, 61-2).

${ }^{8}$ Sobre el punto de expunción véase W. M. Lindsay, Palaeographia Latina III, London 1924, 65-6; B. Bischoff, Latin Palaeography: Antiquity and the Middle Ages, Cambridge 1990, 171.

${ }^{9}$ Los puntos se observan perfectamente en la imagen adjunta, que incluye las siete últimas líneas completas de la primera columna de $\mathrm{N} \mathrm{III} 3^{\mathrm{r}}$ (es el folio 19r): nuestro pasaje comienza en la segunda línea y termina, como es habitual en este manuscrito para ahorrar espacio (De Nonno, La grammatica, 59, n. 6), al final de la primera que se ve en la imagen. 


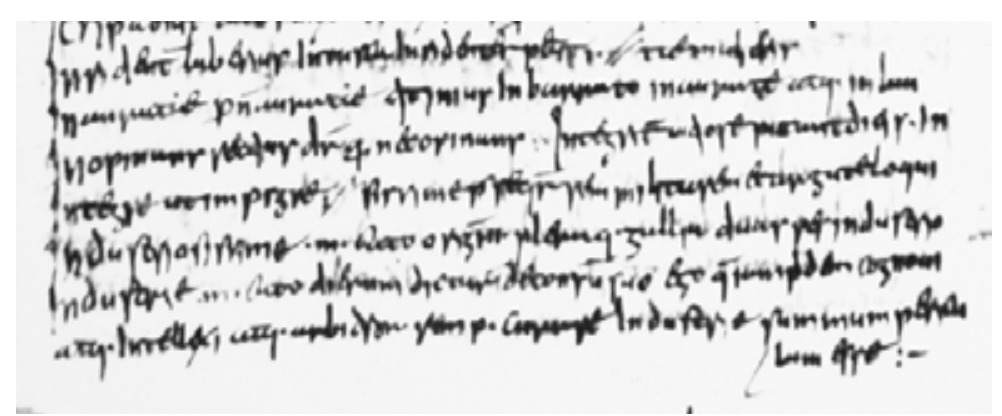

Lámina 1: Fragmento de Neapolitanus IV.A.8, f. 19r

Sin embargo, resulta confuso el modo en que, primero Keil, y, a remolque, Barwick, han reflejado en su aparato crítico la anotación del manuscrito: ese "corr. -te" entre paréntesis provoca confusión, y puede dar a entender una corrección marginal o supra lineam. Es, además, una práctica distinta a la que los mismos editores siguen en situaciones idénticas: así, por ejemplo, cuando en el app. crit. de p. 307, 8 imprimen "priami" (primi en el texto) o en el de p. 297, 7, "sita" (sit en el texto). La confusión aumenta en el caso de Barwick, que, al prescindir de la virgulilla de la $e$ usada por Keil (inauratę), da lugar a un texto verdaderamente confuso: "inaurate (corr. -te)".

$\mathrm{Si}$ de los manuscritos pasamos a las ediciones, observamos que la lectura inaurate pro non aurate ha sido la preferida desde la editio princeps de $1532^{10}$ hasta Barwick en 1925. La acogieron, además de ellos, G. Fabricius $(F$. Sosipatri Charisii Artis grammaticae libri quinque, Basilea 1551,222) y H. Keil (Grammatici Latini, 202). Prefieren, sin embargo, la lectura inauratae pro non auratae, tanto $\mathrm{H}$. van Putschen (Putschius) (Grammaticae Latinae auctores antiqui, Hanau 1605, 181) como F. Lindemann (Corpus Grammaticorum Latinorum ueterum, t. IV, Leipzig 1840, 120), aunque éste último no se resiste a expresar su perplejidad en una nota: "Mirum est, Grammaticum illum locum huc rettulisse et inter adverbiorum exempla posuisse" (Corpus Grammaticorum, 120 n. 1) ${ }^{11}$. En este reparto, resulta especialmente significativa la opción de Putschius, al menos si es cierto, como señala De Nonno ${ }^{12}$, que "è stato possibile dimostrare che, laddove si discosta [scil. Putschius] dal testo del suo predecessore Fabricius, egli testimonia

${ }^{10}$ Esta edición fue realizada por J. P. Cyminius sobre la base del manuscrito Neapolitanus IV.A.9. Puede consultarse una reproducción fotográfica en http://brbl-dl.library.yale.edu/vufind/Record/3478008 (líneas 5-6 del f. 74v).

${ }^{11}$ Merece la pena citar también aquí la anotación de J. H. Neukirch, De fabula togata Romanorum, Leipzig 1833, 103-4: "Inaurate pro non aurate... sic legendum est; non, ut habet Putsch., Inauratae pro non auratae. Nam grammaticus toto hoc capite de adverbiis tantum agit. Quum autem adverbii inaurate exemplum in promptu non haberet, istum Titinii locum posuit". Debemos a un revisor anónimo la referencia a esta obra de Neukirch.

${ }^{12}$ De Nonno, La grammatica, 63, n. 20. 
-salvo pochissime congetture- lezioni di C”i3. Si es así, ello supondría un fuerte argumento a favor de la lectura inauratae, que tendría refrendo en los dos testimonios más conspicuos $(\mathrm{N}$ y $\mathrm{C}$ ) de las dos ramas principales de la tradición de Carisio. Pero, como veremos de inmediato, no es el único ni el más fuerte de los argumentos.

Es evidente que el más sólido apoyo de la lectura inauratae está en el ejemplo de Titinio que da sustento a la entrada; y es que en él la palabra inauratae, obviamente referida a mulieris, no puede ser otra cosa que un adjetivo en genitivo ${ }^{14}$. Debemos, en cambio, explicar por qué un adjetivo merece una entrada en una lista de adverbios. La razón, oculta no ya para los editores, sino también, como hemos podido ver, para la mayor parte de los copistas que intervinieron en la tradición del texto de Carisio, es la siguiente: inauratae no merece su inclusión en la lista por su condición de adjetivo, sino por su preverbio in-. En efecto, una parte de la tradición gramatical latina tipificaba los preverbios como adverbios: esa es, por ejemplo, la doctrina que se deduce de un pasaje de la introducción del propio Julio Romano a su liber de aduerbio, en el que, al señalar las homonimias (consortia) que se establecen entre el adverbio y otras partes orationis, habla de algunos adverbios praepositionibus similia ut pro sub prae propter (Roman.-Char. gramm. p. 247, 8-9), un pasaje en el que esos ejemplos muy probablemente no aparecen en calidad de adverbios propiamente dichos sino más bien como preverbios ${ }^{15}$.

Dato aún más significativo en apoyo de nuestra propuesta es que en la lista de Romano aparezcan dos preverbios, concretamente dis y per, ambos comentados como equivalentes del adverbio ualde: Roman.-Char. gramm. p. 257, 22-4 Dis pro ualde Plautus in Bacchidibus (481), 'nam illa memorare, quae illum facere uidi, / dispudet'; Roman.-Char. gramm. p. 273, 23 Per pro perquam, ualde, ut perduellio perquam duellio.

En fin, como último dato probatorio, señalemos que nuestra explicación no es sólo válida para ratificar el texto inauratae pro non auratae, sino

${ }^{13}$ Es $\mathrm{C}$ el famoso codex deperditus con el que Barwick mejoró sustancialmente el texto de Carisio (K. Barwick, "Zur Geschichte und Rekonstruktion des Charisius-Textes", Hermes 59, 1924, 322-55 y 420-9; Barwick, Flavii Sosipatri, XII; De Nonno, La grammatica, 62-4.

${ }^{14}$ Salvo que se admita la lectura mulieres de C, que, como nos apunta un revisor anónimo, estaría apoyada por listas de insultos a mujeres que se encuentran en Plauto (Plaut. Cist. 415-7 y frg. 97 Lindsay), pero no ha sido acogida por los editores más recientes, ya que Daviault (fr. 7) edita $<->$ inauratae atque inlautae mulieris (véase A. Daviault, Comoedia togata. Fragments, Paris 1981). De todos modos, es sugerente la idea de un influjo de Plauto, que, como nos indica otro revisor anónimo, combina también dos adjetivos en Men. 801 quando te auratam et uestitam bene habet.

${ }^{15}$ En efecto, no son nunca adverbios pro ni $s u b$, y muy rara vez prae (ThLL X 2, 373.55). Sí es, en cambio, frecuente como adverbio propter, cuya presencia aquí tal vez tenga que ver con su uso en el compuesto propterea, dada la discusión suscitada entre los gramáticos respecto a los adverbios compuestos de preposición y el pronombre ea: así en el propio Julio Romano, siguiendo a Helenio Acrón (Roman.-Char. gramm. p. 261, 6-8 Interea loci Terentius... ubi Acron 'quaeritur' inquit 'quo accentu dici debeat interea loci'). 
también para aclarar definitivamente otras dos chocantes entradas nominales, a saber, la que precede inmediatamente a la que hemos discutido aquí (Roman.Char. gramm. p. 262, 29 Inopinans rectius dicitur quam necopinans), y otra que aparece unas páginas más arriba (Roman.-Char. gramm. p. 257, 16-8 Decolorem pro sine colore Cicero Tuscularanum II, cum Herculem daret ingemescentem: 'iam decolorem sanguinem omnem exorbuit'). Huelga decir que su presencia en la lista de adverbios la deben esos adjetivos/ participios a los preverbios in-, nec- y de- ${ }^{16}$. De ellos, sólo el primero le mereció a Lindemann (Corpus Grammaticorum, 120, n. 2) un comentario de extrañeza: "Hic quoque locus non huc pertinere videtur, cum exemplum non sit adverbii". Pero ninguno de los dos fue sometido, como el de inauratae, a enmienda, tal vez porque la corrección no resultaba tan sencilla como la que suponía convertir un diptongo ae en $e$.

En definitiva, el repaso histórico de esta simple confusión ha dejado al descubierto una interesante doctrina gramatical, minoritaria ${ }^{17} \mathrm{y}$, en cierto modo, latente, cuyo origen puede ser interesante rastrear, aunque ello cae ya fuera de los límites de esta nota crítica.

${ }^{16}$ En rigor, tal como nos apunta un corrector anónimo, sólo los casos de inopinans y necopinans constituyen un paralelo exacto al de inauratae, ya que -dice- el resto de los preverbios citados pueden utilizarse como praepositiones separatae (tal es, ciertamente, el caso de de- y per-, pero no el de dis-). Aunque la distinción de preposiciones y preverbios es conocida y aplicada por Julio Romano en el capítulo de praepositione (por ejemplo, en Roman.-Char. gramm. p. 308, 3-5) no parece ser tenida en cuenta aquí, pues unas y otros se clasifican indistintamente como adverbios, seguramente porque la fuente seguida está inmersa en otra tradición teórica, como inmediatamente apuntaremos.

${ }^{17}$ Que era en efecto minoritaria lo demuestra un texto de Prisciano (Prisc. gramm. III 505, 22-3) sobre la preposición inter, de la que nos dice que plerique Latinorum praepositionem accipiunt, Graeci autem aduerbium. 